\title{
Design of miniature multi - function chassis suspension device
}

\author{
Tingyv Wang ${ }^{1, ~ a}$, Xiaorong $L v^{1, b^{*}}$ \\ ${ }^{1}$ Sichuan Agricultural Uniersity, Ya'an, China 46 new road, Sichuan, No. 625014 \\ a964026857@qq.com, 'blxrxj2008@163.com
}

Keywords: Miniature multi-function chassis. Suspension device. Three point convergence. Design. Abstract. This design uses three point convergence type suspension system, The upper pull rod adopts the hydraulic oil cylinder to adjust the buried angle, and use rope winding device connection the lower pull rod, It can independently adjust the working condition of the equipment. Small multi-function chassis suspension device is designed for small agricultural power equipment design, especially solve suspend ploughshare problem of the buried angle adjustment, realize the real-time adjustment of the buried angle, thus limiting the depth of the plow and improve the quality of cultivated land.

\section{Introduction}

Traditional small tractor suspension device is very heavy, structure is complex, occupying a large space, and large operation energy consumption, when the hanging plow and plough fields, plow buried angle regulation is through the screw rod adjusting that is not convenient and tedious [1]. Therefore, this design provides a small multi-function chassis suspension device, the utility model has the advantages of simple structure, light weight, low energy consumption, efficient use of space, easy to operation of the driver and with plough hanging use, through the control upper pull rod and the oil cylinder can at any time convenient adjustable plow the buried angle, suitable for mechanization of cultivated land, In addition, the driver can easily operate the lifting and falling of the hanging machine by winding the rope mechanism, thereby effectively reducing the labor intensity and improving the production efficiency and the quality of production.

The structure and working principle of the miniature multi - function chassis suspension device

\section{Integral structure design of suspension device}

Suspension device composed of rope winding mechanism, upper support seat, longitudinal bracket, an oil cylinder, a lower pull rod component, and an operator controls the hydraulic oil valve switch used to control the hydraulic cylinder work,connecting the lower pull rod through the winding mechanism, operated winding mechanism, so that the upper and lower pull rod together to complete the field work needs. The overall structural design of the suspension device is shown in figure 1: 


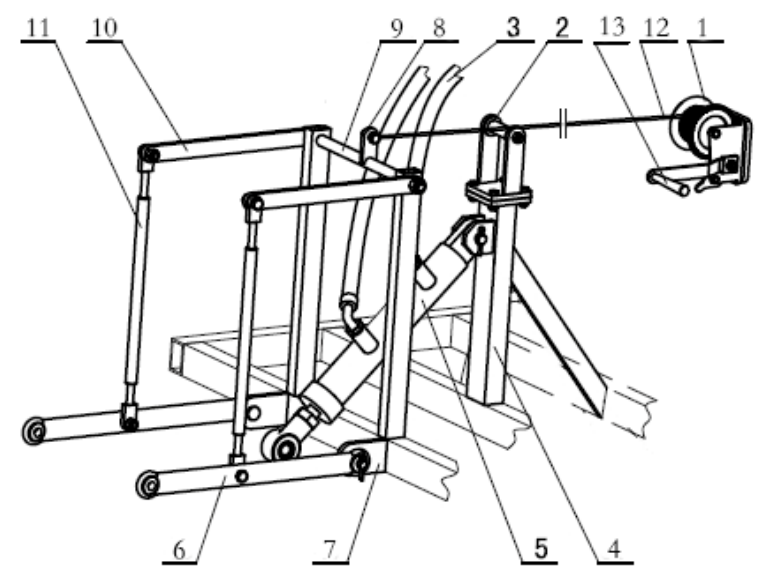

Fig. 1 Structure of suspension device

1.Rope winding mechanism; 2. Upper support seat; 3. Tubing; 4. Longitudinal bracket; 5.Oil cylinder; 6.Lower pull rod; 7. Lower pull rod support seat; 8. Power plate;9. Rotating shaft; 10. Pull rod; 11. Regulating rod; 12. Wire rope; 13. Handle.

\section{Working principle of miniature multi function chassis suspension device:}

The design of the suspension plough as an example.

Implementation of lift the suspension of the ploughshare, by the operator shaking around the handle 13 of the rope mechanism 1 , then wire rope 12 forward tension winding to rope winding mechanism 1, wire rope 12 pulling forward power plate 8 around the rotating shaft 9 center line forward turned an angle, pull rod 10 also with rotating shaft 9 turned an angle and with back-end regulating rod 11 upward movement. At the same time, in regulating rod 11 to sports, lower pull rod 6 will be connected with the lower pull rod support seat 7 pin as a fulcrum to rotate, thereby driving the hanging connected plough in the lower pull rod lift. Conversely, the handle back to shake, the steel wire rope is loosened, lifting mechanism by the weight of its own gravity and plowshare back to its original position, ploughshare decline and fall to the ground.

Adjust the angle that between ploughshare buried angle of ploughshare and the ground level, move the multi-function chassis cab hydraulic valve, oil cylinder 5 activities end extends out of the front end of the plough lift, the buried angle decreases, reduced tillage depth; when backward drive hydraulic valve, then oil cylinder 5 retraction, the front of the plough to fall, buried angle becomes larger and larger, increase deep tillage, through regulating repeatedly until it reaches deep tillage requirements and farming to complete tillage operations.

\section{Design of main working parts}

In this paper, the design of the main working parts of the suspension device is through the virtual three-dimensional design. In the light of on the basis of the structure scheme and main technical parameters of the suspension device, the functional requirements and characteristics of each component, the structure design of the components are designed by using UGNX software[2].

\section{Virtual design of the lower pull rod mechanism}

In order to ensure that the lower pull rod mechanism has good control performance, a lower pulling rod mechanism is developed, which has the advantages of simple structure, small occupation space, flexible and reliable operation, etc..

Under the two ends of the lower pull rod with ball head sleeve, and the hexagonal bolt is connected with the rack, frame using welding is fixed at the end of the tractor chassis. The power plate and the pull rod are fixedly connected with the rotating shaft. The two ends of the regulating 
rod are respectively connected with the pull rod and the lower pull rod by six angle bolts. The structure of the lower pull rod mechanism is shown in figure 2.

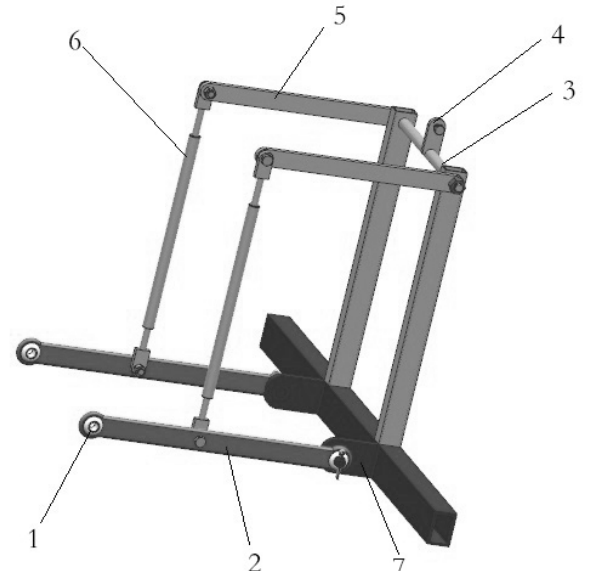

Fig. 2 Lower pull rod mechanism

1. ball head sleeve; 2 . Lower pull rod;

3. Rotating shaft; 4. Power plate;

5. Pull rod 6. Regulating rod;

\section{Lower pull rod support seat.}

\section{Virtual design of the upper pull rod mechanism}

The upper pull rod mechanism adopts hydraulic cylinder as the rod, the upper support seat and longitudinal bracket connected by six angle bolts. The longitudinal bracket and the supporting plate are fixedly connected with the vehicle body through the welding method. After calculation, the inner diameter of the cylinder is $20(\mathrm{~mm})$, the diameter of the piston is $8(\mathrm{~mm})$, the piston stroke is $160(\mathrm{~mm})$, the cylinder length is $220(\mathrm{~mm})$, the section pressure is $7(\mathrm{Mpa})$. The structure of the upper pull rod mechanism is shown in Figure 3.

\section{Virtual design of rope winding mechanism}

The rope winding mechanism is to be more efficient and rapid in the process of lift agricultural implements. The rope winding mechanism is composed of a fixed pulley, a self- locking mechanism, a handle and a steel wire rope, and the steel core wire rope with a nominal diameter of $12(\mathrm{~mm})$ and a tensile strength of $1570(\mathrm{Mpa})$ and a minimum breaking tension of $80.5(\mathrm{KN})$ are obtained by the test. Its final determination is $6 \times 19 W+F C$ structure [3]. Its structure is shown in Figure 4

The location of the installation should take into account the operator's convenience and practicality, so it will be installed in the bottom right of the tank bracket . as shown in Figure 5. 


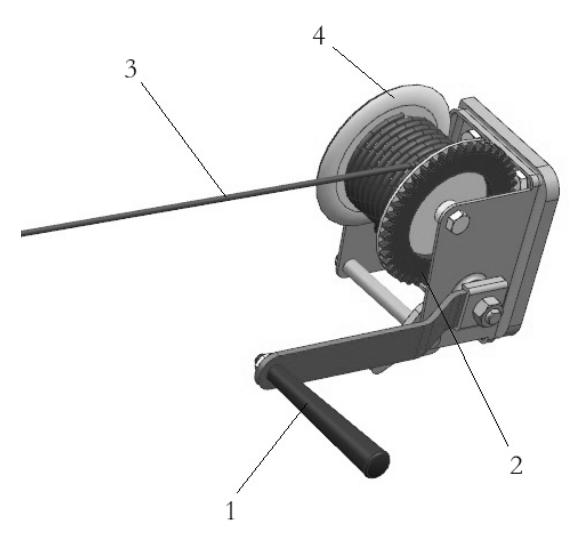

Fig. 4 Rope winding mechanism

1. Handle; 2. self-locking mechanism;

3. Wire rope; 4. Fixed pulley;

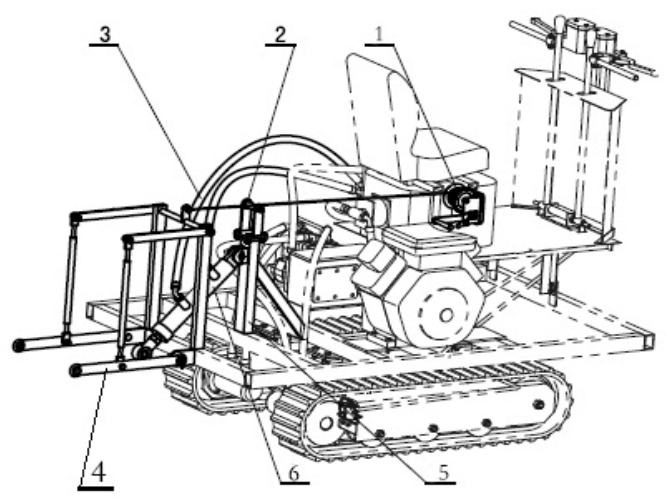

Fig. 5 Integral assembly drawing

1. Rope winding mechanism;

2. Upper support seat; 3. Tubing;

4. Lower pull rod; 5. Longitudinal bracket;

6. Oil cylinder;

\section{Conclusions}

The overall structure of the design is novel, It has a strong practicality. In view of the suspension system of small multi - function site tractor, the lifting of the work equipment can be effectively realized in the range of allowable load. The device has been successfully applied to the national utility model patent.In practical operation, the work is stable. The design is suitable for the combination of small tractors in hilly areas, high reliability. It will promote the development of agricultural machinery.

\section{Acknowledgements}

The study was supported by the National Natural Science Foundation of China (51105261)

Correspondence Author: LvXiaorong, College of Information \& Engineering, Sichuan Agricultural University, Ya'an, China, 1xrxj2008@163.com.

\section{References}

[1] Hongxin Liu, Wenzhe Li. Study of automobile and tractor[M]. Beijing: China Agriculture Press. 2013 (08).In Chinese.

[2] Xueyan Jia, Jia Tu UGNX9 complete self-study manual[M]. Beijing: People's Posts and Telecommunications Publishing House,2014 (08). In Chinese.

[3] chen Ling. Purchase and use of steel wire rope[J]. Metal products.2004 (05).In Chinese. 